\title{
Unexpected Selectivity of UV Light Activated Metal-Oxide-Semiconductor Gas Sensors by Two Different Redox Processes
}

\author{
Wenting Li and Gu Xu \\ Department of Materials Science and Engineering, McMaster University, Hamilton, ON, Canada L8S 4L7
}

Correspondence should be addressed to Gu Xu; xugu@mcmaster.ca

Received 27 November 2015; Revised 3 February 2016; Accepted 14 February 2016

Academic Editor: Sang Sub Kim

Copyright (C) 2016 W. Li and G. Xu. This is an open access article distributed under the Creative Commons Attribution License, which permits unrestricted use, distribution, and reproduction in any medium, provided the original work is properly cited.

\begin{abstract}
The conflict between the two existing models was resolved, to provide a clear explanation for the unexpected "selectivity" found in UV light activated metal-oxide-semiconductor (MOS) gas sensors during the detection of reducing agents. A new model based on the dynamic equilibrium of adsorbed oxygen concentration was constructed by two types of responses: (1) when the MOS surface is adsorbed with oxygen, the conductance of the sensor increases upon injection of reducing agents (RA) ( $\alpha$-type) and (2) when the MOS surface is not covered by oxygen, the conductance decreases upon injection of RA ( $\beta$-type). The proposed model was verified by the experiments of $\mathrm{ZnO}$ based MOS gas sensors, to reveal the origin of the unexpected "selectivity" found by the optimum intensity, where the current drop, due to the reaction between RA and MOS, which increases with UV power and levels with the reciprocal background current, which decreases with the UV power.
\end{abstract}

\section{Introduction}

In the past few decades, metal-oxide-semiconductor (MOS) systems have been investigated intensively for the purpose of gas sensing [1-3]. MOS sensors are usually operated at elevated temperatures typically in the range of $200-400^{\circ} \mathrm{C}$ [4]. This impedes the detection of flammable, explosive gases, and biospecies. To overcome this limit, Saura [5] developed the UV activated $\mathrm{SnO}_{2}$ films to detect trichloroethylene and acetone vapor at room temperature, which has been followed by many other research groups to investigate the mechanism. In particular, de Lacy Costello et al. [4], Alenezi et al. [6], and Comini et al. [7] found that, for the detection of reducing agents (RA), by tuning the intensity of the UV light, a maximum sensitivity can be observed for each analyte under a different intensity of UV light, indicating that it is possible to achieve the desirable "selectivity" of the MOS sensors by varying the UV light intensity. This is unexpected, however, since the change in intensity only alters the number of photons, but not the photon energy, which is given by the product of the Planck constant and the speed of light over the wavelength of the photon, and does not change as long as the wavelength remains the same. Thus the variation in intensity will not affect the energy states in the MOS gas sensor. Several theoretic models [8-10] were proposed to discuss the selection mechanisms involved. But no satisfactory results have been found so far. Therefore a new mechanistic model needs to be developed to reveal the origin of the unexpected "selectivity" here.

Moreover, two conflicting mechanistic schemes have been established in the previous literature. Gong et al. [11, 12] claimed that the reducing agents (RAs) consume charge carriers and thus decrease the conductivity of the sensing materials. But other groups $[6,13,14]$ found that upon injection of RA the electrons would be released back to the conduction band of the MOS, leading to the drop of the barrier height and increase of the mobility and conductivity of the sensing materials. Therefore, the new model should also be able to resolve the conflict and to accommodate the findings involved.

Therefore, it is the purpose of this paper to investigate these problems. Through the analysis, it is found that, by varying the amount of adsorbed oxygen on the MOS sensor surface, the RA reactions can be switched between two types: 


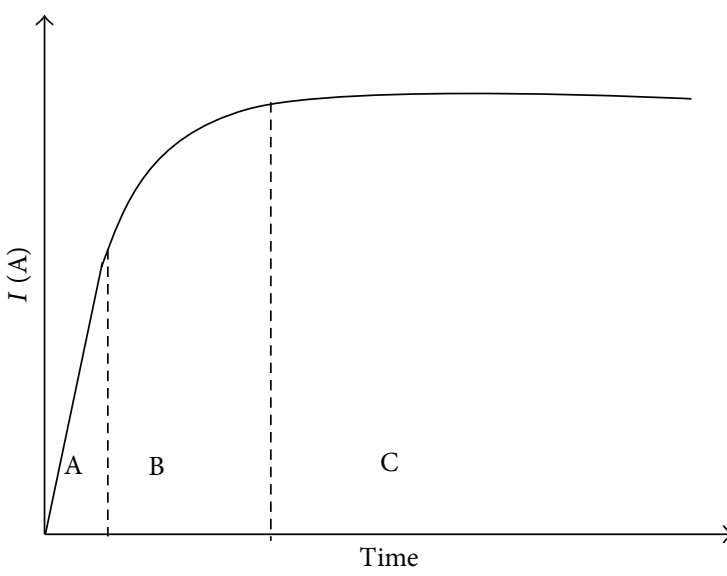

(a)
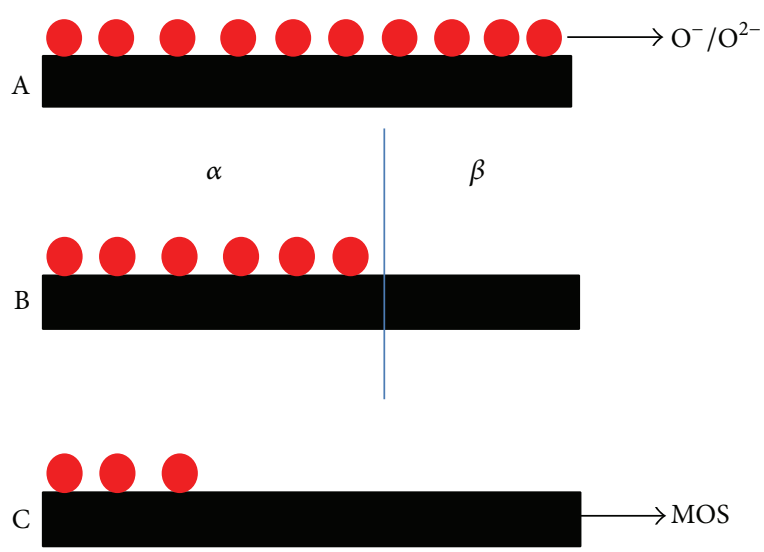

(b)

FIGURE 1: (a) Schematic illustration of the current response of a MOS sensor under UV irradiation. (b) Schematic illustration of the adsorbed oxygen species on a MOS sensor surface, where the red circles represent adsorbed oxygen.

the carrier receiving ( $\alpha$-type) and the carrier consuming ( $\beta$ type). Based on the analysis and related experimental observations, a new model is established to resolve the conflict and to reveal the origin of the unexpected "selectivity."

\section{Results and Discussion}

In a usual n-type MOS sensor, environmental oxygen species are adsorbed on the sensor surface and receiving electrons from the MOS. These trapped negative charges cause an upward band bending and an electron-depleted region to reduce the conductance. When the sensor is under UV light, with photon energy larger than the band gap, the holes created in the MOS will migrate from the bulk to the surface and react with the adsorbed oxygen species, and the electrons coproduced will, however, be repelled by the negatively charged oxygen species and thus released back to the conduction band of the semiconductor. As a result, the conductance will increase, as shown in Figure 1(a), for the typical current response of a MOS sensor under the UV exposure, where there are three regions: $(A)$ is the fast-rise region, $(\mathrm{B})$ the slow-rise region, and $(\mathrm{C})$ the constant region. The region $(A)$ is attributed to the generation of electron-hole pairs upon excitation of UV light, which increases the carrier density (electrons and holes). The region (B) is due to the hole migration from the bulk to the surface and recombination reaction between the adsorbed oxygen species. In particular, holes will recombine with $\mathrm{O}^{1-}$, which was found to be the dominant species in the UV excited sensors, rather than $\mathrm{O}^{2-}$, more popular under thermal activation [6]. (B) is much slower than $(\mathrm{A})$, since the time needed for a chemical reaction is much longer than that of electronic process. And region (C) is when the concentration of adsorbed oxygen reaches a dynamic equilibrium in an atmospheric environment (depletion saturated).

Correspondingly, the schematic concentrations of adsorbed oxygen species in three regions are described in
Figure 1(b). Because the carrier generation process takes place much faster than the desorption of oxygen species, the latter can be neglected in region (A), where the surface is dominated by oxygen adsorption. As the time goes on, however, in region (B) a gradual decrease of oxygen concentration occurs, until an equilibrium concentration is reached in the atmospheric environment (C). Two types of surfaces can thus be found on the MOS; $\alpha$ is where the MOS surface is adsorbed with oxygen species, and $\beta$ is without. When a reducing agent (RA) is introduced, the reaction that takes place in region $\alpha$ is simply the chemical reaction between the RA and adsorbed oxygen ions [6]. For example, the reactions involving ethanol become [6]

$$
\begin{aligned}
& \mathrm{CH}_{3} \mathrm{CH}_{2} \mathrm{OH}+\mathrm{O}^{-}{ }_{\text {(ads) }} \longrightarrow \mathrm{C}_{2} \mathrm{H}_{4} \mathrm{O}+\mathrm{H}_{2} \mathrm{O}+1 \mathrm{e}^{-} \\
& \mathrm{CH}_{3} \mathrm{CH}_{2} \mathrm{OH}+\mathrm{O}^{2-}{ }_{(\text {ads })} \longrightarrow \mathrm{C}_{2} \mathrm{H}_{4} \mathrm{O}+\mathrm{H}_{2} \mathrm{O}+2 \mathrm{e}^{-}
\end{aligned}
$$

where the electrons are released from oxygen ions and going back to the conduction band of MOS. Therefore the conductivity or photocurrent will increase, for there are decreased band bending/barrier heights. This process can be associated to "carrier receiving" ( $\alpha$-type) process of the MOS. On the other hand, the reactions in region $\beta$ occur between the MOS and the RA directly. According to Li et al. [12], these reactions consume both holes and excited electrons, which lead to the decrease of the conductivity or photocurrent. And this process can be termed as "carrier consuming" ( $\beta$-type) process. The two processes can be incorporated to form a new model; if the reaction is dominated by $\alpha$ region, the overall current increases. Conversely, it can be argued that when there is a current decrease, it must be dominated by $\beta$ or the carrier consuming reaction. Since the $\beta$-type takes less amount of time than that of $\alpha$-type, the two processes will usually be separated in the time variation of current. In any case, since the adsorbed oxygen concentration can be altered by UV light intensity or exposure time, the photocurrent may 
be either increased or decreased, as found in the literature, depending on the competition of the two processes $[6,11-14]$.

Based on the above, it is possible to obtain an optimum intensity for an analyte (RA); that is, the "selectivity" mechanism can thus be revealed by the new model. Since the overall signal is given by the (current change)/(the background) or (current change) * (reciprocal background), it is desirable to take the current change when a stable background current is achieved, or when the depletion is saturated after long time UV exposure ( $C$ in Figure 1). Therefore, the signal is usually based on the current drop by $\beta$ process, rather than $\alpha$-type, which takes longer time. Since various RAs react with MOS differently, due to the reducing ability of the RAs under UV light, the current drops are different. At the qualitative level, it can be expected that stronger RA gives a larger current change (drop) than the weaker, which can be further illustrated by Figure 2, where the current changes increase due to the increased MOS surface by UV exposure.

Moreover, these RA curves will eventually level off at higher UV power, due to the limited number of gas molecules. Combining the fact that the reciprocal background current is inversely related to the UV power, but without divergence due to the dark current, the signals will be maximized at a certain UV power, where the two reached the same level, as shown in Figure 2 by the dashed curves. In particular, for strong RAs, it only needs a lower amount of UV light to reach the peak signal, whereas for the analyte with weaker reducing ability, it will require stronger UV intensity to achieve the same. Therefore, the difference in the reducing ability of RAs under UV light will lead to a variable optimum UV intensity, to achieve the observed "selectivity." In the meantime, with changing analyte concentration, the optimum intensity value remains the same. This can be argued by the saturation of the RA on the MOS surface, since the amount of desorption of oxygen species will not change under variable analyte concentrations, when the saturation is reached. Therefore, not only the maximum sensitivity under an optimum UV power but also a correlation established between the optimum UV intensity and the reducing ability of RAs can be explained.

As the signal maxima have been well observed (e.g., [4]), experiments will be designed only to verify the new model by changing the adsorbed oxygen concentration on the MOS surface and then observing the photocurrent change upon acetone injection. The former may easily be achieved by annealing of the MOS sensors in oxygen atmosphere, which enhances chemisorbed oxygen [15], or by long time UV light exposure, which reduces the chemisorbed oxygen.

From Figure 3, it is evident that photo current changes dramatically, switching between two completely opposite trends, upon varying the adsorbed oxygen. For example, before annealing, corresponding to low oxygen adsorption, the current drops ( $\beta$-type) upon RA injection (Figures 3(a) and $3(\mathrm{~b})$ ), whereas after annealing, corresponding to high oxygen adsorption, the current increases drastically ( $\alpha$-type) upon RA injection (Figures 3(c) and 3(d)) (the initial quick drop is attributed to the still exposed MOS surface, which induces the same $\beta$-type, and is quickly overwhelmed by $\alpha$-type). Moreover, the experiments were repeated under

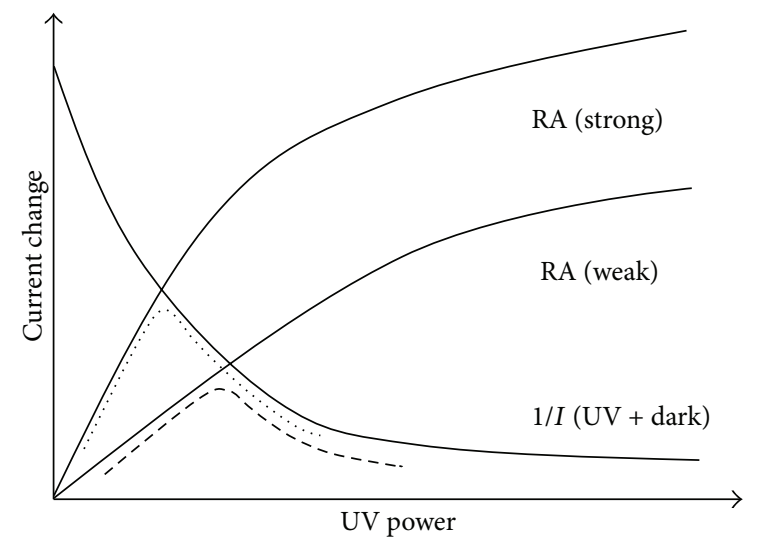

FIGURE 2: Schematic representation of the influence of UV light intensity on the RAs reducing ability, leading to the peak signals under variable UV power.

different ambient conditions, also shown in Figure 3, where the current responses of the $\mathrm{ZnO}$ film sensor were compared under two different environments: $20^{\circ} \mathrm{C}$ and $45 \% \mathrm{RH}$ and $22^{\circ} \mathrm{C}$ and $55 \% \mathrm{RH}$. It can be found that although there are quantitative differences in sensitivity $\left(\sim 13.2 \%\right.$ at $20^{\circ} \mathrm{C}, 45 \%$ $\mathrm{RH}, \sim 18.9 \%$ at $22^{\circ} \mathrm{C}, 55 \% \mathrm{RH}$ before annealing; $\sim 22.2 \%$ at $20^{\circ} \mathrm{C}, 45 \% \mathrm{RH}, \sim 23.1 \%$ at $22^{\circ} \mathrm{C}, 55 \% \mathrm{RH}$ after annealing), the current change directions remain the same upon RA injection.

To further demonstrate the photo current switching trends under variable amount of surface oxygen, Figure 4 compares the photo current responses of three different surface oxygen states; (a) before annealing, where small amount of oxygen species was adsorbed on the sensor surface, the photo current decreases upon the injection of acetone, indicating $\beta$-type, carrier consuming process, or RA reacting directly with MOS. The sensitivity, measured by the current variation over the background, was about $10.8 \%$. (b) After annealing, the background current dropped by almost an order of magnitude, caused by the space charge layer introduced by ionized adsorbates and increased barrier heights [16] and larger amount of adsorbed oxygen. In the meantime, sharp increase of the photocurrent can be observed after the injection of acetone, indicating $\alpha$-type, carrier receiving process, or RA reacting with oxygen, quickly after some $\beta$ type. The sensitivity also increased to about $33.8 \%$. (c) After 24 hours of oxygen desorption by the UV light, the oxygen was removed, and the process reverses back to the situation of (a); that is, the current drops upon RA injection but increases upon injection of nonreactive gas. In the meantime, the background current due to the MOS bulk is not recovered by the UV exposure. Therefore, a conclusion can be drawn that the variation in adsorbed oxygen on MOS surface indeed switches the reaction types between the carrier receiving $(\alpha)$ and carrier consuming $(\beta)$, which is supported by the experimental results from the UV light activated MOS gas sensors. 

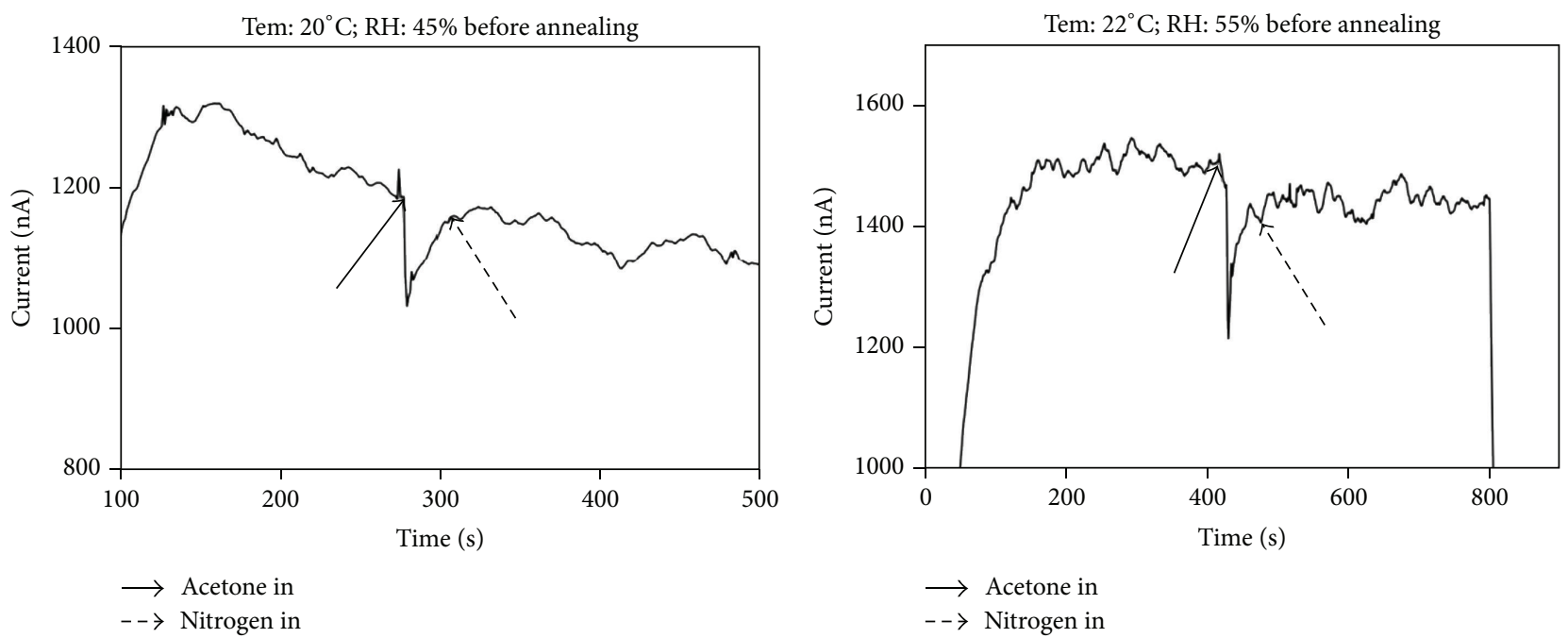

(a)

(b)

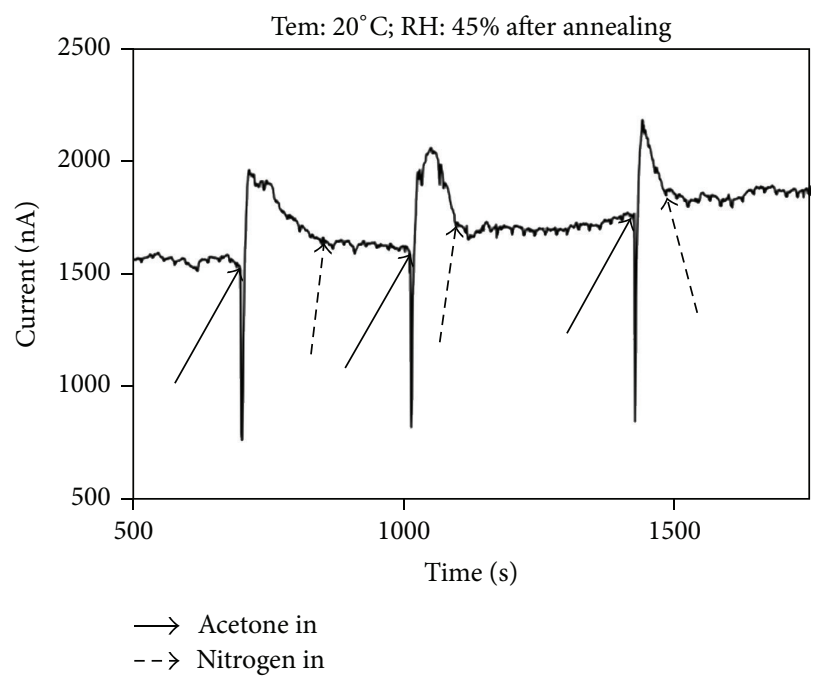

(c)

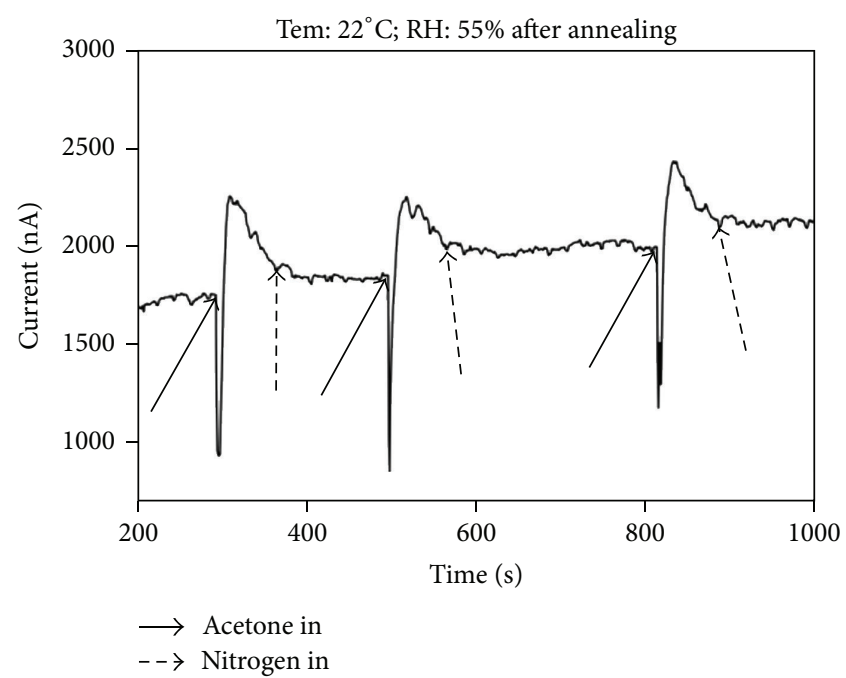

(d)

Figure 3: Current responses of the $\mathrm{ZnO}$ film sensor to acetone vapor under $\mathrm{UV}$ irradiation: (a) current response before annealing under $20^{\circ} \mathrm{C}$ and $45 \%$ relative humidity. (b) Current response before annealing under $22^{\circ} \mathrm{C}$ and $55 \%$ relative humidity. (c) Current response after annealing under $20^{\circ} \mathrm{C}$ and $45 \%$ relative humidity. (d) Current response after annealing under $22^{\circ} \mathrm{C}$ and $55 \%$ relative humidity. The solid arrows mark the injection of RA and dashed arrows the injection of nitrogen, serving as a nonreactive gas.

\section{Conclusion}

To summarize, a new model based on the equilibrium of two competing processes has been developed, to reveal the "selectivity" mechanism of UV activated MOS gas sensors for reducing agents. The conflict between the two existing models found in literature has been resolved and unified by the new model as well. The maximum intensity is obtainable by the combination of both the current drop, due to the reaction between RA and MOS, which increases with UV power, and the reciprocal background current, which decreases with the UV power. They were verified experimentally by changing the amount of adsorbed oxygen species on the sensor surface, when the photocurrent change is reversed in direction as predicted here.

\section{Experimental}

Nanoparticles of zinc oxide (99.9\%, APS $87 \mathrm{~nm}$, specific surface area $15-25 \mathrm{~m}^{2} / \mathrm{g}$ ) were obtained from Aldrich Chemical Co. The substrate is shown in Figure 5. Two ITO stripes are placed on a glass substrate and connected to the electrodes of the testing system. The zinc oxide MOS material is placed on top of the two stripes and constitutes the electrical circuit. The ITO electrodes were also replaced by Au and Ag in the repeated measurements, when identical results were reproduced.

The zinc oxide nanopowder was placed in an agate pestle and mortar and a certain amount of distilled water was added to form a thick slurry [4]. Then $3 \mathrm{~mL}$ of the zinc oxide paste 


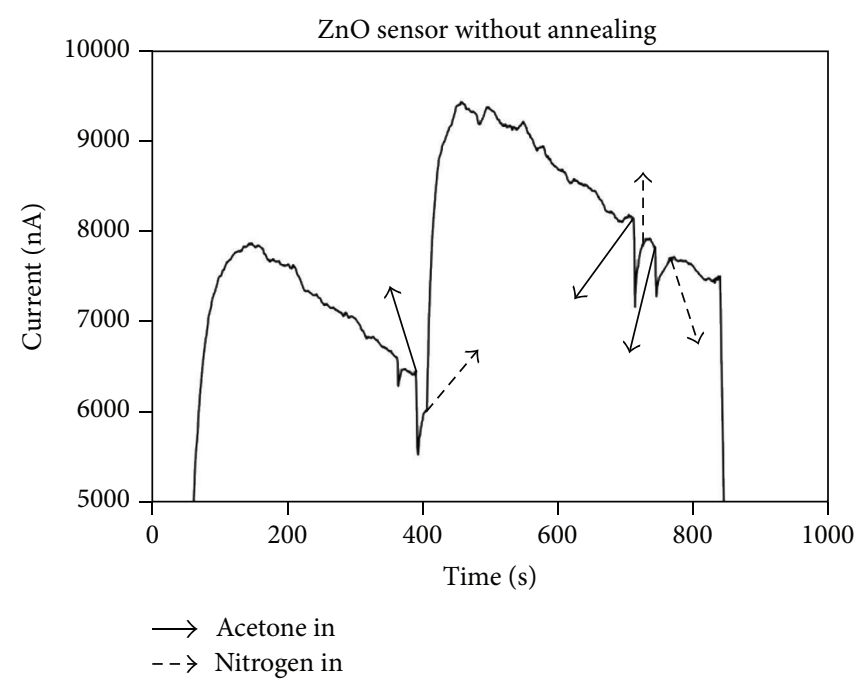

(a)

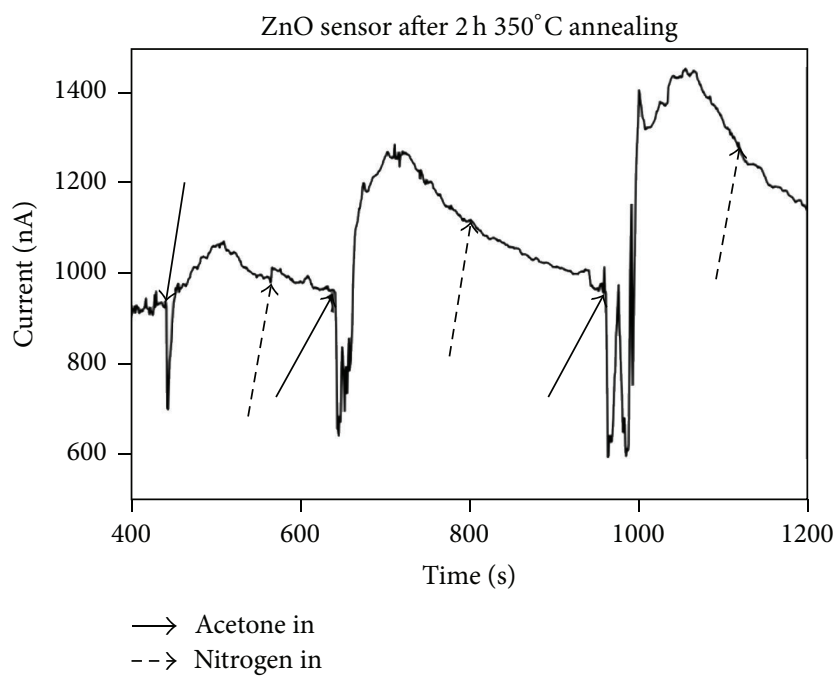

(b)

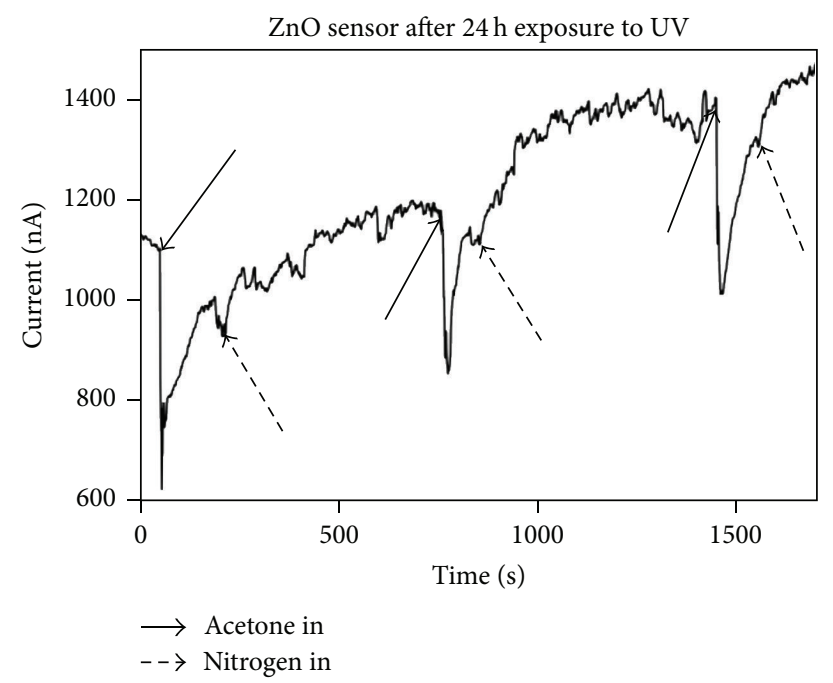

(c)

FIgURE 4: Current responses of the $\mathrm{ZnO}$ film sensor to acetone vapor under UV irradiation: (a) current response before annealing. (b) Current response after annealing under $350^{\circ} \mathrm{C}$ for two hours. (c) Current response of the annealed sample after 24 hours of UV light treatment. The solid arrows mark the injection of RA and dashed arrows the injection of nitrogen, serving as a nonreactive gas.

was added on the substrate to form a thin film. The sample was dried in room temperature and atmosphere for 24 hours.

The sensor performance was then tested by a Pico Ampere Meter (Keithley 485) as shown in Figure 5. The whole apparatus set was put in a $1 \mathrm{~m}^{3}$ chamber with air atmosphere. After each test, the chamber was flushed with pure Nitrogen to make sure there was no acetone remaining. The voltage applied to the sensor was $10 \mathrm{~V}$.

The back of the sensor was attached to a fixed point on a white board. A high intensity UV lamp (B-100AP UV lamp, Co. UVP) that peaks at $365 \mathrm{~nm}$ was placed 15 inches away from the sample to provide an intensity about $3 \mathrm{~mW} / \mathrm{cm}^{2}$. The test gas was provided by a nitrogen tank containing $200 \mathrm{ppm}$ acetone. The UV light was turned on for 5 minutes before the injection of the acetone to gain a relatively stable baseline.
The same sample must be used for the three conditions: before annealing, after annealing, and after $24 \mathrm{~h}$ of UV light treatment. The sensitivity $S$ of the sensor is defined as $\mid I_{0}-$ $I_{g} \mid / I_{0}$, where $I_{g}$ is the current of the sensor in gas and $I_{0}$ is the current of the sensor in air before injection.

\section{Conflict of Interests}

The authors declare no conflict of interests.

\section{Authors' Contribution}

Wenting Li carried out the sample preparation and measurements and wrote the paper. Dr. Gu Xu supervised the project and helped in revising the paper. 


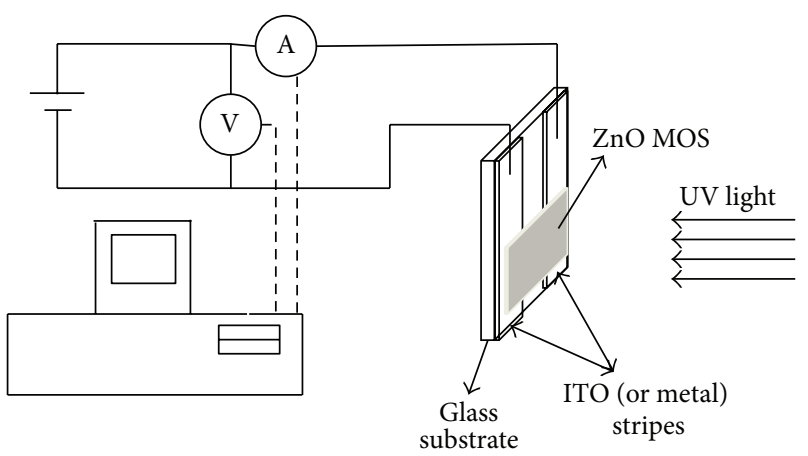

Figure 5: Schematic diagram of the sensor testing system.

\section{Acknowledgments}

The authors would like to thank Dr. Adrian Kitai and Dr. Tony Petric for providing the experimental equipment. This work was funded by Natural Science and Engineering Foundation of Canada.

\section{References}

[1] Q. Qi, T. Zhang, L. Liu et al., "Selective acetone sensor based on dumbbell-like $\mathrm{ZnO}$ with rapid response and recovery," Sensors and Actuators B: Chemical, vol. 134, no. 1, pp. 166-170, 2008.

[2] G. Kiriakidis, M. Suchea, S. Christoulakis, and N. Katsarakis, "High performance gas sensing materials based on nanostructed metal oxide films," Reviews on Advanced Materials Science, vol. 10, no. 3, pp. 215-223, 2005.

[3] H.-W. Zan, C.-H. Li, C.-C. Yeh, M.-Z. Dai, H.-F. Meng, and C.-C. Tsai, "Room-temperature-operated sensitive hybrid gas sensor based on amorphous indium gallium zinc oxide thinfilm transistors," Applied Physics Letters, vol. 98, no. 25, Article ID 253503, 2011.

[4] B. P. J. de Lacy Costello, R. J. Ewen, N. M. Ratcliffe, and M. Richards, "Highly sensitive room temperature sensors based on the UV-LED activation of zinc oxide nanoparticles," Sensors and Actuators B: Chemical, vol. 134, no. 2, pp. 945-952, 2008.

[5] J. Saura, "Gas-sensing properties of $\mathrm{SnO}_{2}$ pyrolytic films subjected to ultrviolet radiation," Sensors and Actuators B: Chemical, vol. 17, no. 3, pp. 211-214, 1994.

[6] M. R. Alenezi, A. S. Alshammari, K. D. G. I. Jayawardena, M. J. Beliatis, S. J. Henley, and S. R. P. Silva, "Role of the exposed polar facets in the performance of thermally and UV activated $\mathrm{ZnO}$ nanostructured gas sensors," The Journal of Physical Chemistry C, vol. 117, no. 34, pp. 17850-17858, 2013.

[7] E. Comini, L. Ottini, G. Faglia, and G. Sberveglieri, " $\mathrm{SnO}_{2}$ RGTO UV Activation for CO Monitoring," IEEE Sensors Journal, vol. 4, no. 1, pp. 17-20, 2004.

[8] S. Mishra, C. Ghanshyam, N. Ram, R. P. Bajpai, and R. K. Bedi, "Detection mechanism of metal oxide gas sensor under UV radiation," Sensors and Actuators B: Chemical, vol. 97, no. 2-3, pp. 387-390, 2004.

[9] X. Wang, S. S. Yee, and W. P. Carey, "Transition between neckcontrolled and grain-boundary-controlled sensitivity of metaloxide gas sensors," Sensors and Actuators B. Chemical, vol. 25, no. 1-3, pp. 454-457, 1995.
[10] J. D. Prades, R. Jimenez-Diaz, M. Manzanares et al., "A model for the response towards oxidizing gases of photoactivated sensors based on individual $\mathrm{SnO}_{2}$ nanowires," Physical Chemistry Chemical Physics, vol. 11, no. 46, pp. 10881-10889, 2009.

[11] J. Gong, Y. Li, X. Chai, Z. Hu, and Y. Deng, "UV-light-activated $\mathrm{ZnO}$ fibers for organic gas sensing at room temperature," Journal of Physical Chemistry C, vol. 114, no. 2, pp. 1293-1298, 2010.

[12] Y. Li, J. Gong, G. He, and Y. Deng, "Enhancement of photoresponse and UV-assisted gas sensing with Au decorated $\mathrm{ZnO}$ nanofibers," Materials Chemistry and Physics, vol. 134, no. 2-3, pp. 1172-1178, 2012.

[13] S.-W. Fan, A. K. Srivastava, and V. P. Dravid, "Nanopatterned polycrystalline $\mathrm{ZnO}$ for room temperature gas sensing," Sensors and Actuators B: Chemical, vol. 144, no. 1, pp. 159-163, 2010.

[14] Y. Zhang, B. Liu, D. Wang, Y. Lin, T. Xie, and J. Zhai, "Photoelectric properties of $\mathrm{ZnO} / \mathrm{Ag}_{2} \mathrm{~S}$ heterostructure and its photoelectric ethanol sensing characteristics," Materials Chemistry and Physics, vol. 133, no. 2-3, pp. 834-838, 2012.

[15] S. Major, A. Banerjee, and K. L. Chopra, "Annealing studies of undoped and indium-doped films of zinc oxide," Thin Solid Films, vol. 122, no. 1, pp. 31-43, 1984.

[16] D. H. Zhang and D. E. Brodie, "Effects of annealing ZnO films prepared by ion-beam-assisted reactive deposition," Thin Solid Films, vol. 238, no. 1, pp. 95-100, 1994. 


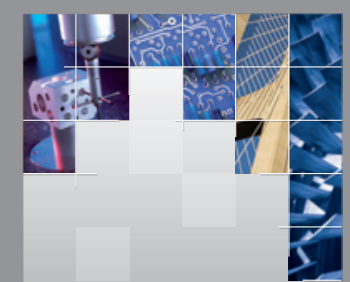

\section{Enfincering}
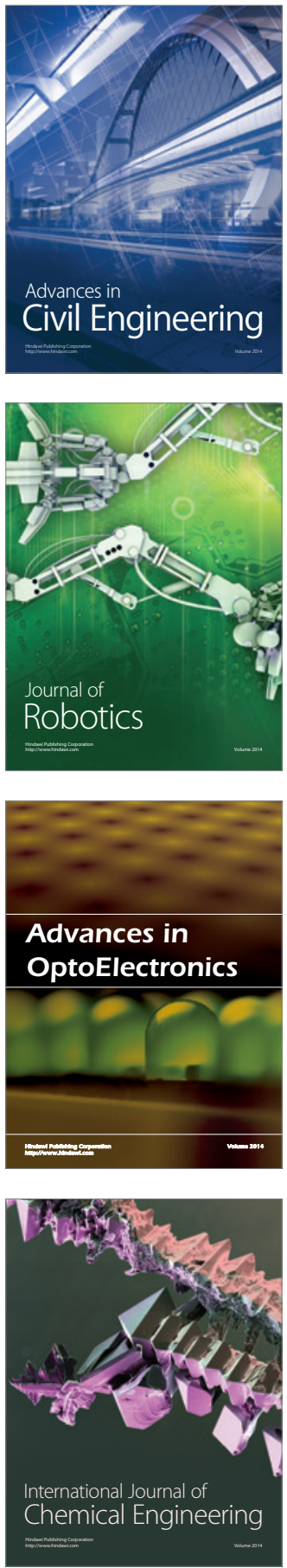

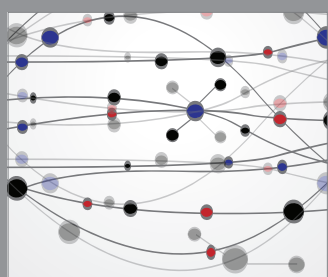

The Scientific World Journal

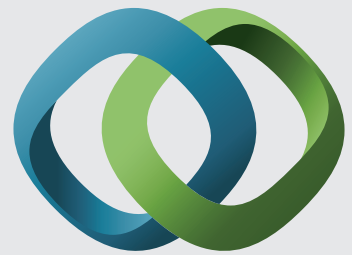

\section{Hindawi}

Submit your manuscripts at

http://www.hindawi.com
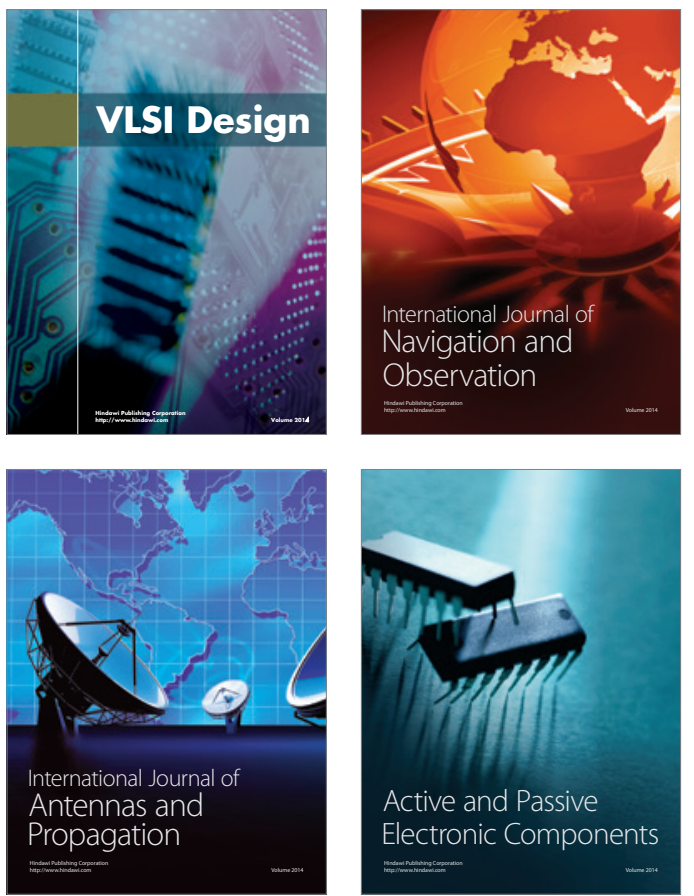
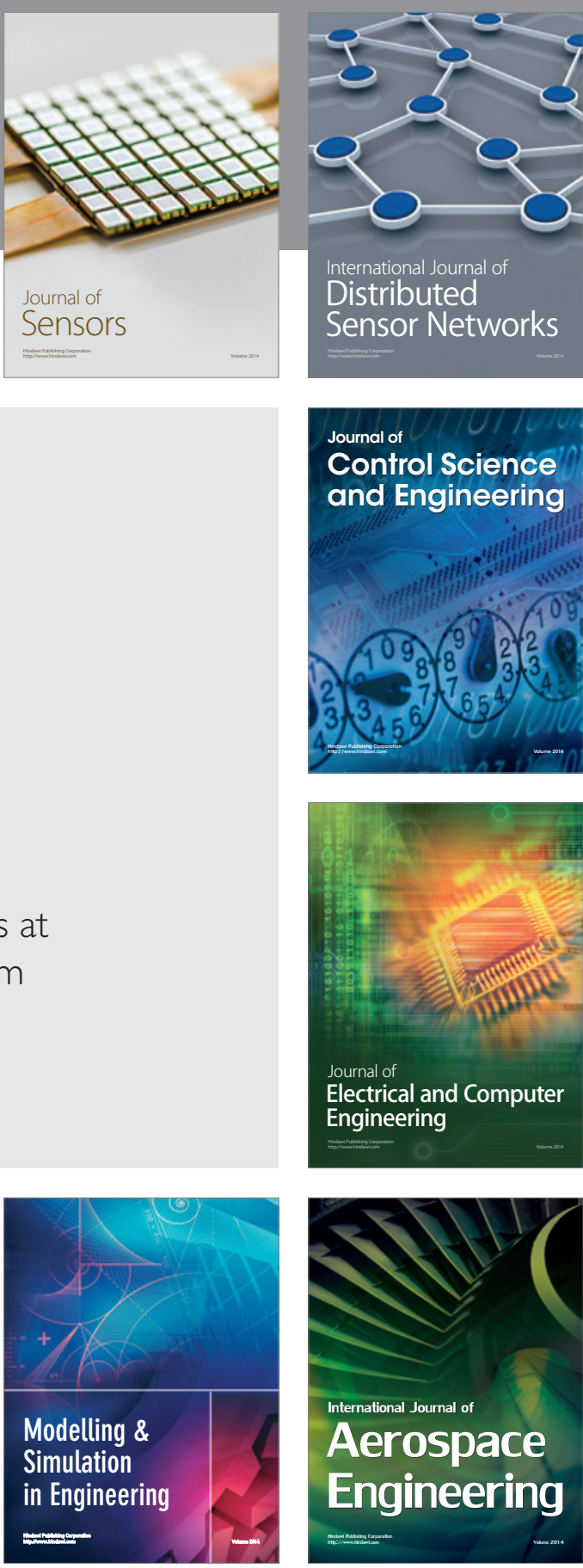

International Journal of

Distributed

Sensor Networks

Journal of

Control Science

and Engineering
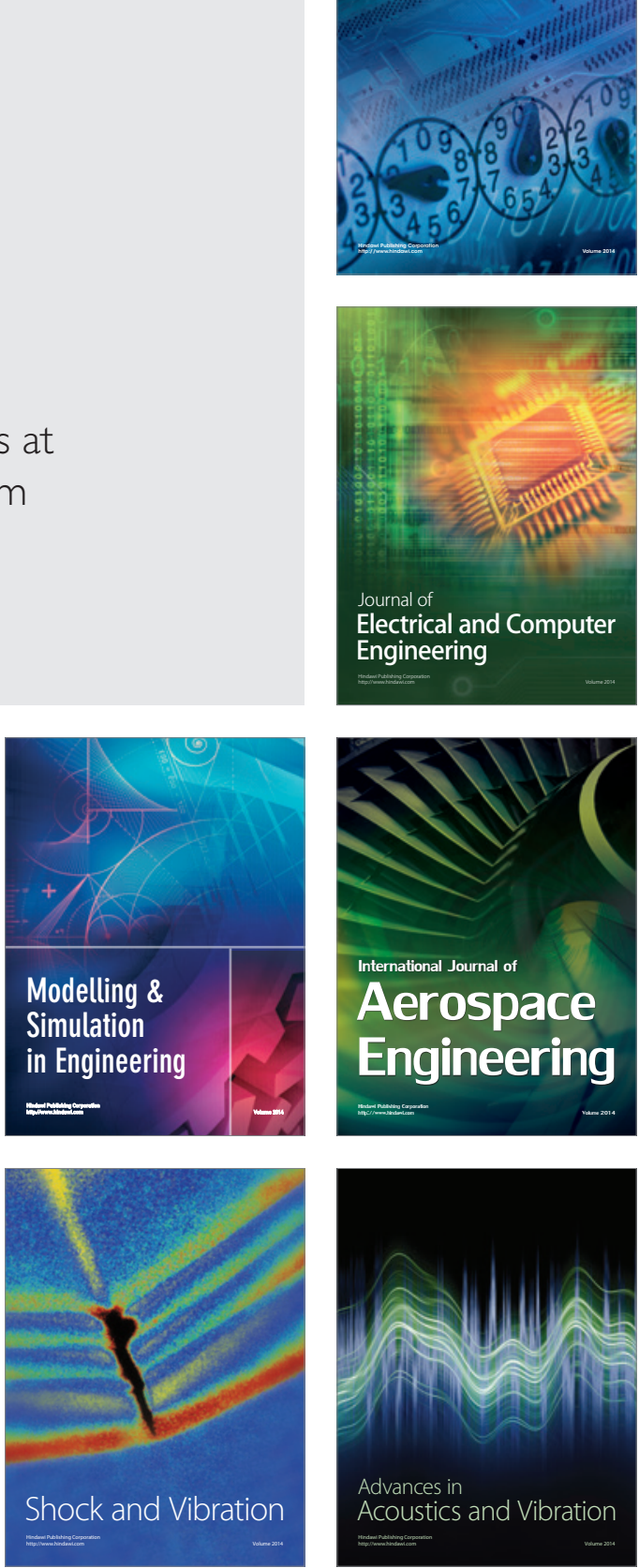